\title{
Dual-Emissive Metal-Organic Framework as a Fluorescent "Switch" for Ratiometric Sensing of Hypochlorite and Ascorbic Acid
}

Yong-Nian Zeng, ${ }^{\dagger}{ }^{\ddagger}$ He-Qi Zheng, ${ }^{\dagger,}$ Jia-Fang Gu, ${ }^{\S}$ Gao-Juan Cao, Wan-E Zhuang, Jian-Di Lin,

${ }^{\dagger}$ Rong Cao, ${ }^{*, *}$ and $\mathrm{Zu}-\mathrm{Jin} \operatorname{Lin} *^{\dagger},{ }^{\ddagger}$

${ }^{\dagger}$ Department of Applied Chemistry, College of Life Sciences, Fujian Agriculture and Forestry University, Fuzhou, Fujian 350002, People's Republic of China.

"State Key Laboratory of Structural Chemistry, Fujian Institute of Research on the Structure of Matter, Chinese Academy of Sciences, Fuzhou, Fujian 350002, People's Republic of China.

${ }^{\S}$ Department of Chemical Engineering, Zhicheng College, Fuzhou University, Fuzhou, Fujian 350002, People's Republic of China.

*Author to whom correspondence may be addressed: rcao@fjirsm.ac.cn; linzujin@fafu.edu.cn; 


\section{1- Chracterization}

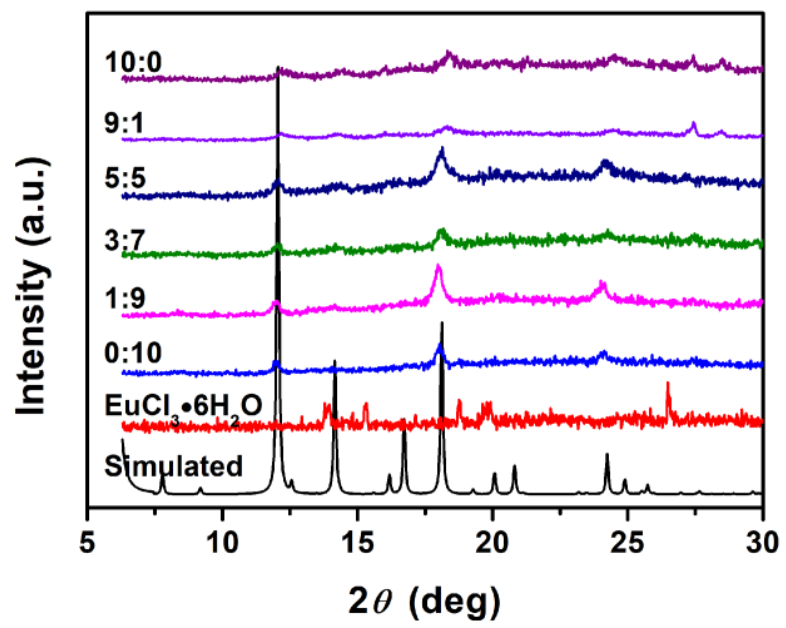

Figure S1. PXRD patterns of multivariate Eu/BPyDC@MOF-253-NH2.

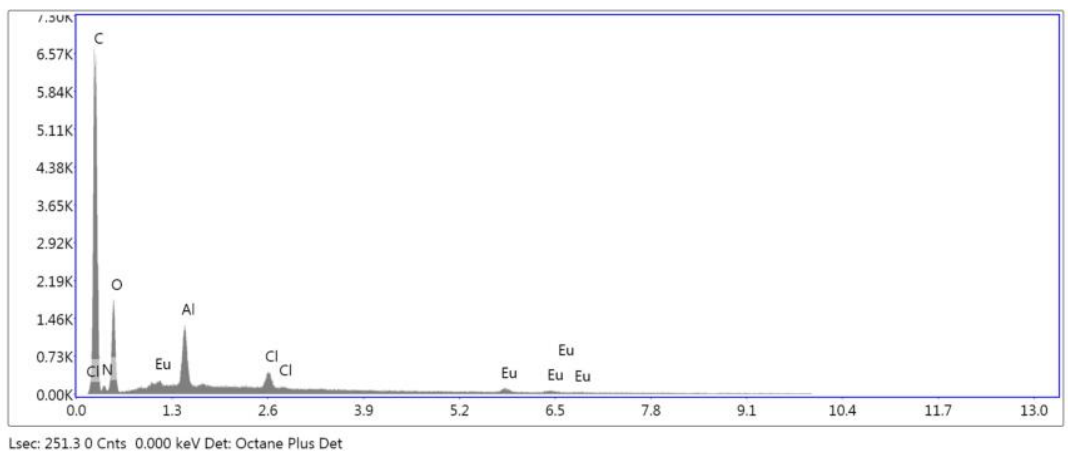

Figure S2. The EDS pattern of 5-5-Eu/BPyDC@MOF-253-NH2.

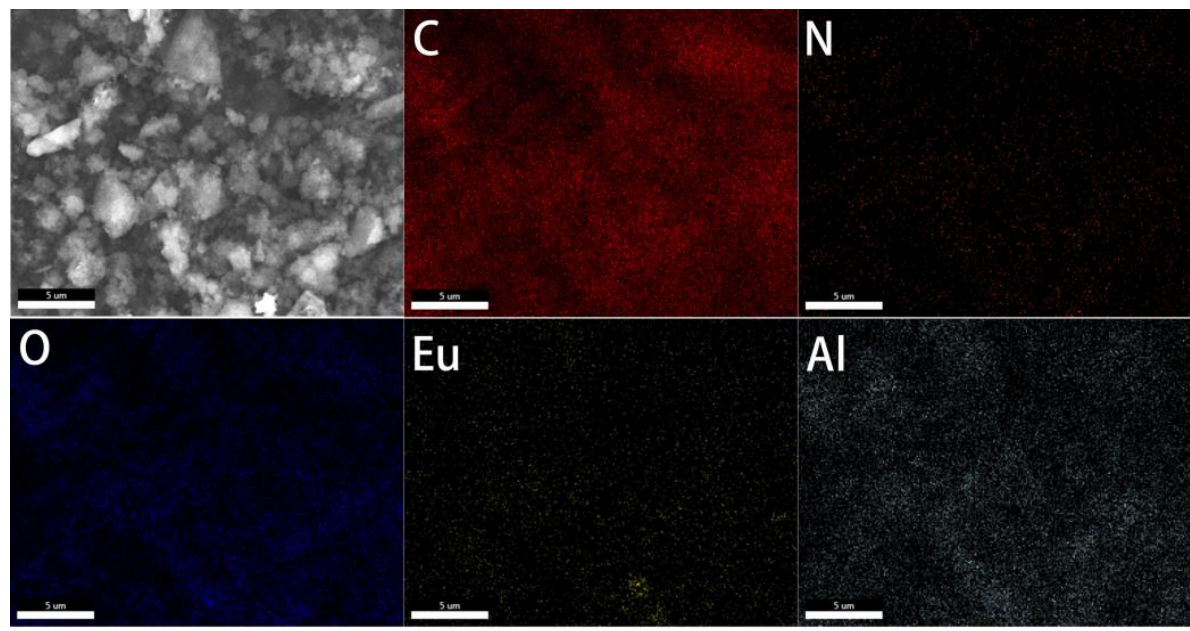

Figure S3. Elemental mapping patterns of 5-5-Eu/BPyDC@MOF-253-NH2. 


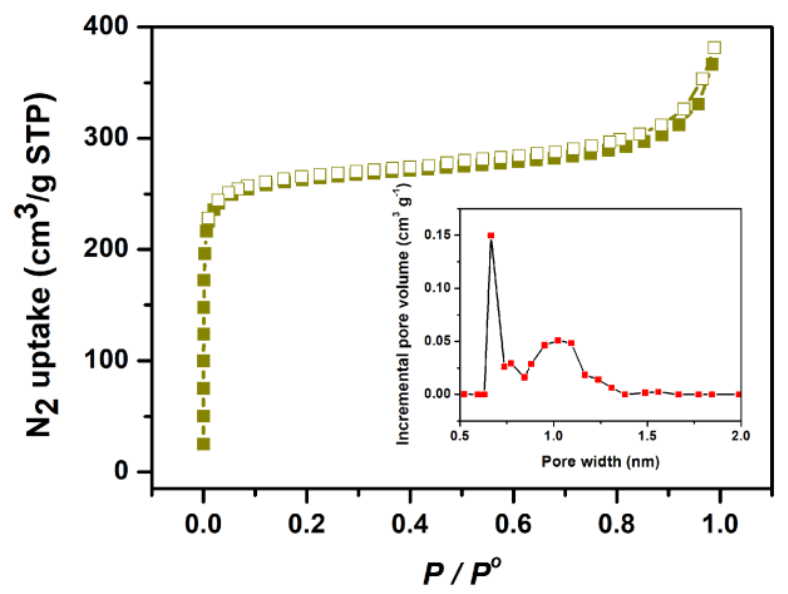

Figure S4. $\mathrm{N}_{2}$ sorption adsorption of the proposed nanosensor (inset: the pore size distribution derived from the $\mathrm{N}_{2}$ adsorption calculated by DFT model).

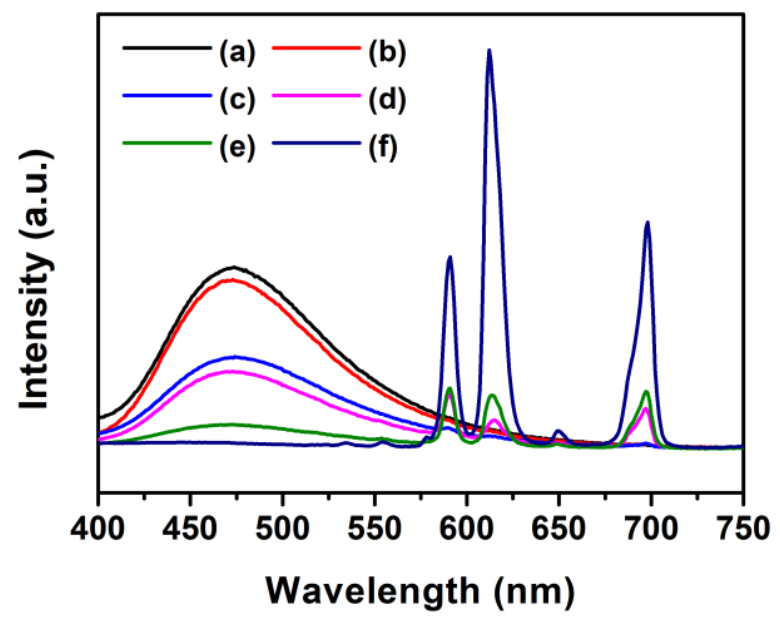

Figure S5. Fluorescent spectra of multivariate (a) 0-10-, (b) 1-9-, (c) 5-5-, (d) 7-3-, (e) 9-1-, and (f) 10-0-Eu/BPyDC@MOF-253-NH2 suspensions in Tris-HCl buffer solution (10 mM, pH =4) containing $20 \mu \mathrm{M}$ of $\mathrm{NaClO}$ when excited at $305 \mathrm{~nm}$. 


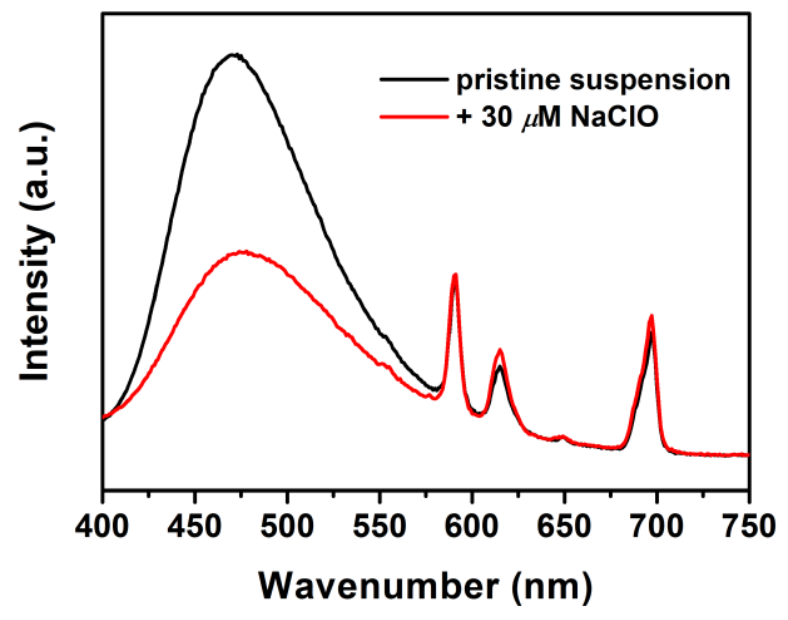

Figure S6. Fluorescent spectra of 5-5-Eu/BPyDC@MOF-253-NH 2 suspension before (black) and after (red) the addition of $30 \mu \mathrm{M} \mathrm{NaClO}$.

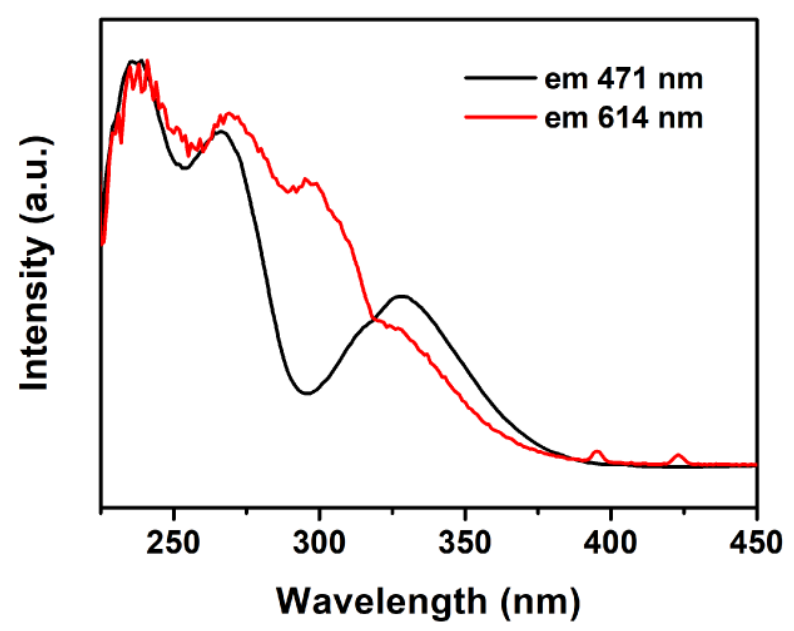

Figure S7. Excitation spectra of 5-5-Eu/BPyDC@MOF-253-NH2 suspension when the emission spectra were monitored at 471 and $614 \mathrm{~nm}$, respectively. 


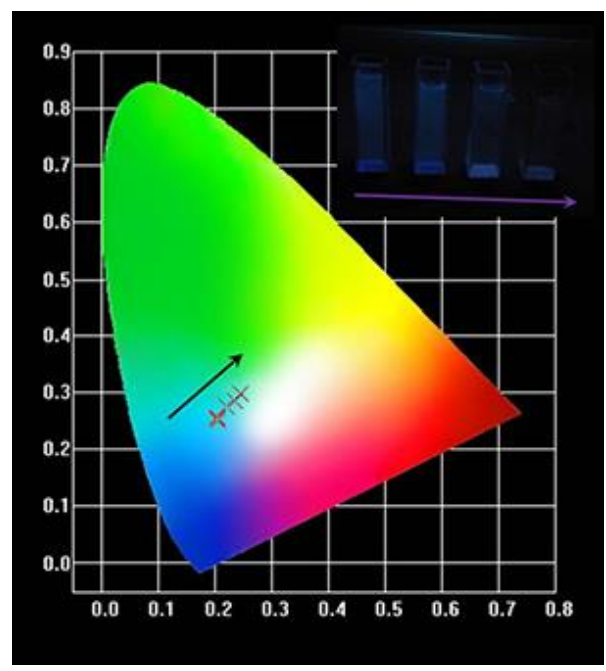

Figure S8. CIE chromaticity diagram of 5-5-Eu/BPyDC@MOF-253-NH2 suspensions with various concentration of $\mathrm{NaClO}(0-30 \mu \mathrm{M})$ (inset: photographs of the suspensions under illumination at $365 \mathrm{~nm}$ ).

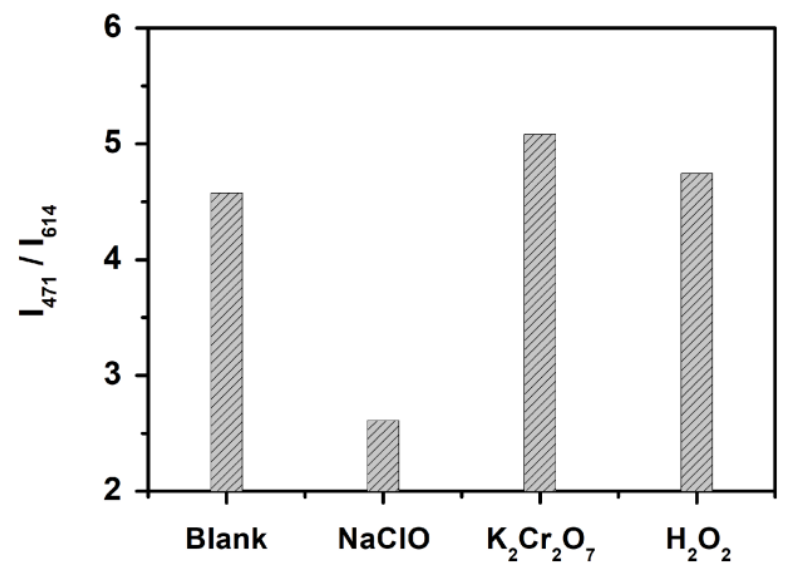

Figure S9. The fluorescent response of 5-5-Eu/BPyDC@MOF-253- $\mathrm{NH}_{2}$ suspensions after adding various oxidants $(60 \mu \mathrm{L}, 1 \mathrm{mM})$. 


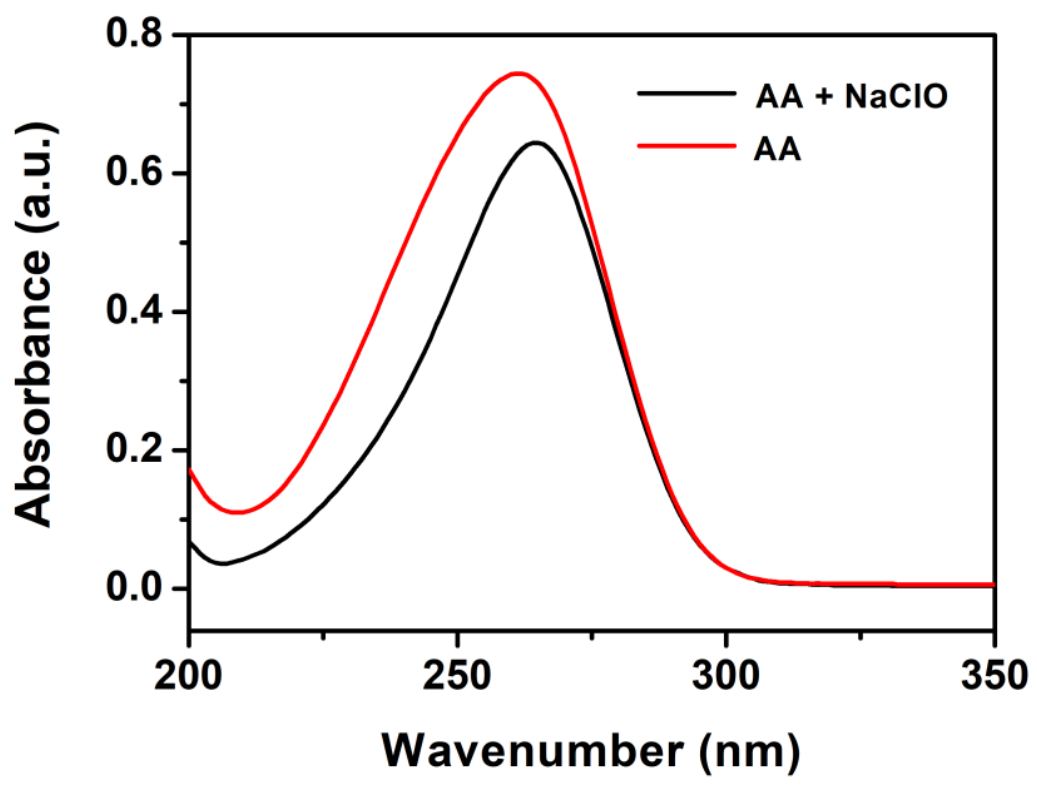

Figure S10. The UV-Vis absorbance spectra of AA $(100 \mu \mathrm{M})$ in Tris-HCl buffer solution $(\mathrm{pH}=4)$ before (red) and after (black) adding $60 \mu \mathrm{L}$ of $\mathrm{NaClO}$ aqueous solution $(1.5 \mathrm{mM})$.

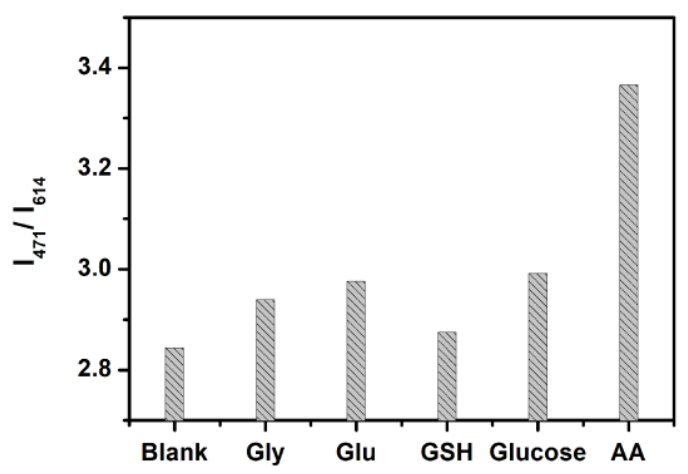

Figure S11. Fluorescence response of the proposed sensing system in Tris- $\mathrm{HCl}$ buffer solution toward various reductants. 


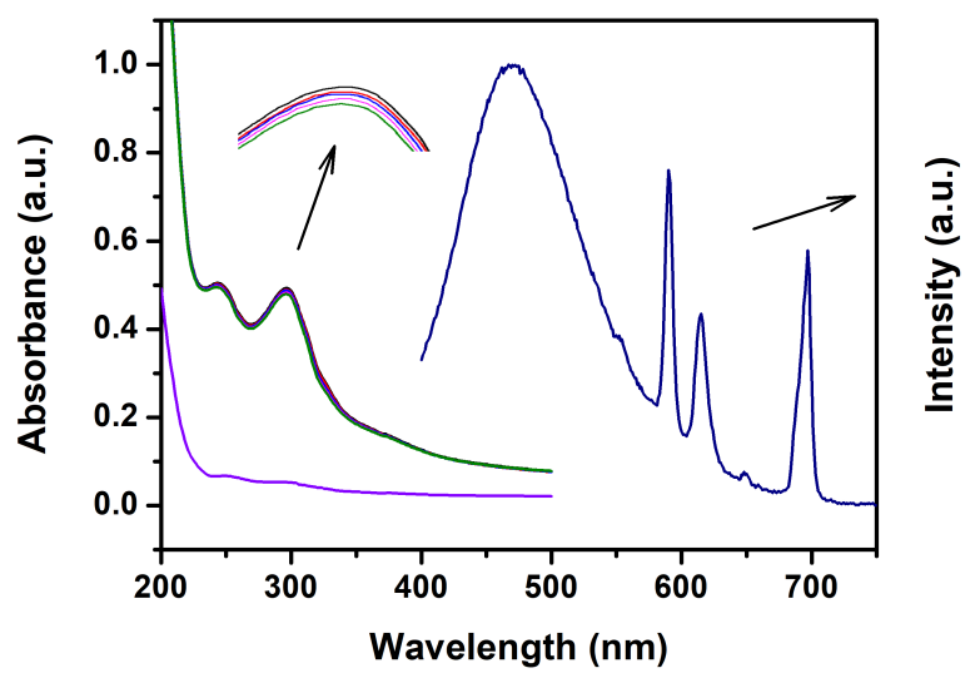

Figure S12. The UV-Vis absorbance spectra of 5-5-Eu/BPyDC@MOF-253- $\mathrm{NH}_{2}$ suspension (black), and the suspension after the addition of $60 \mu \mathrm{L} 0.1 \mathrm{mM}$ (red), 60 $\mu \mathrm{L} 0.5 \mathrm{mM}$ (red), $60 \mu \mathrm{L} 0.5 \mathrm{mM}$ (blue), $60 \mu \mathrm{L} 1.0 \mathrm{mM}$ (purple), and $60 \mu \mathrm{L} 1.5 \mathrm{mM}$ (olive) of $\mathrm{NaClO}$, respectively; the fluorescence of 5-5-Eu/BPyDC@ MOF-253- $\mathrm{NH}_{2}$ suspension after the addition of $60 \mu \mathrm{L} 1.5 \mathrm{mM} \mathrm{NaClO}$ (navy) (the fluoresce intensity was normalized to 1.)

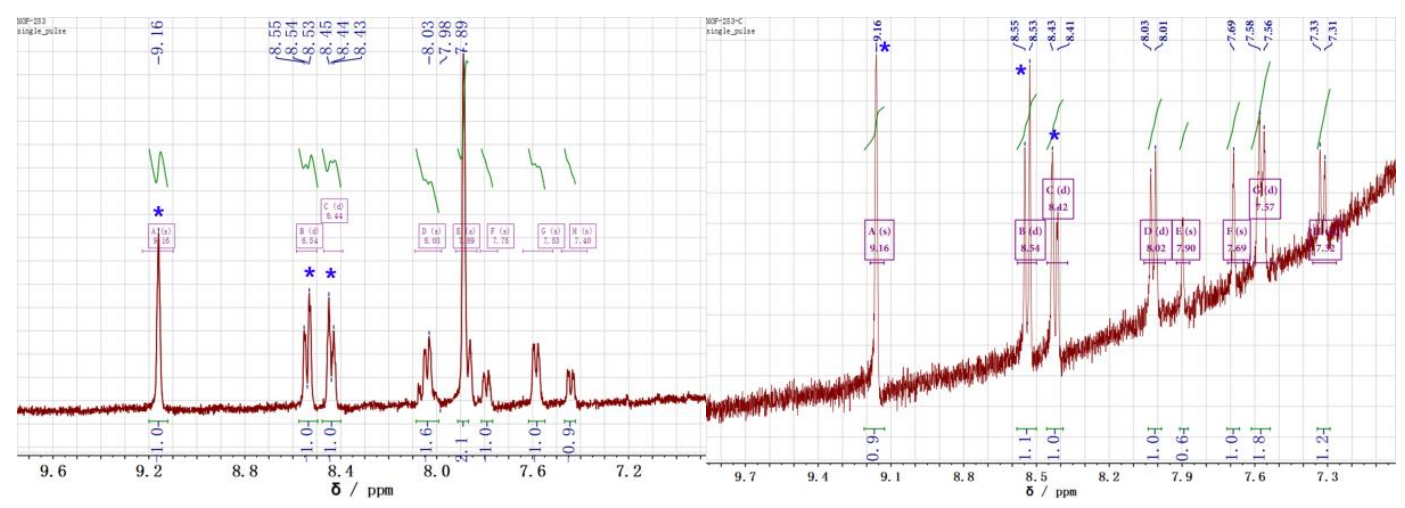

Figure S13 ${ }^{1} \mathrm{H}$ NMR spectra of digested 5-5-Eu/BPyDC@MOF-253-NH2 (left) and its recycled sample (right); the solid samples were digested by $10 \mu \mathrm{L} \mathrm{HF}$ in $d 6$-DMSO; * the peaks were assigned to BPyDC ${ }^{2-}$ ligands. 




Figure S14. The fluorescent decays of 5-5-Eu/BPyDC@MOF-253-NH2 suspensions (containing with $30 \mu \mathrm{M} \mathrm{NaClO}$ ) after the addition of 0 , 100, 200, and $300 \mu \mathrm{L} \mathrm{AA} \mathrm{(1}$ $\mathrm{mM})$, respectively.
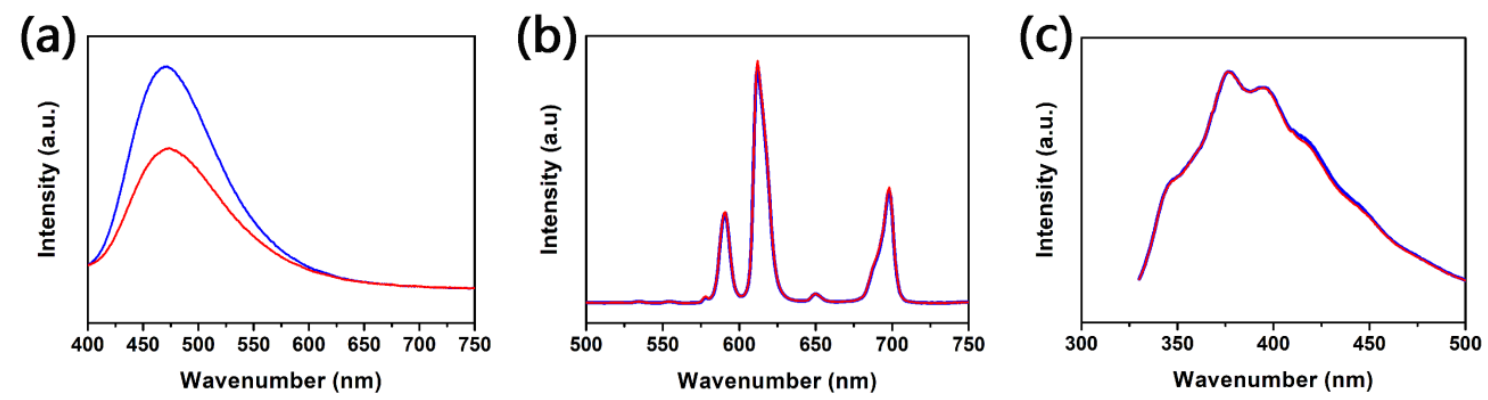

Figure S15. The fluorescent spectra of (a) 0-10-Eu/BPyDC@ MOF-253-NH2 (i.e. DUT-NH2), (b) 10-0-Eu/BPyDC@MOF-253-NH2 (i.e., Eu@MOF-253), and (c) DUT-5 suspensions before (blue) and after (red) the addition of $60 \mu \mathrm{L} \mathrm{NaClO}(1.5$ $\mathrm{mM})$.
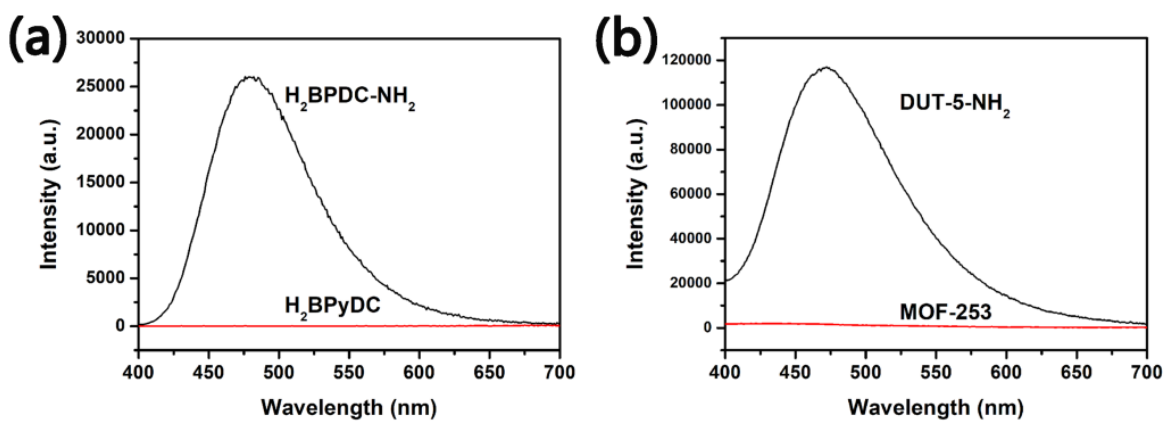

Figure S16. (a) The fluorescence of $\mathrm{H}_{2} \mathrm{BPDC}-\mathrm{NH}_{2}$ and $\mathrm{H}_{2} \mathrm{BPyDC}$ ligands in DMSO 
solution (20 ppm) when excited at $305 \mathrm{~nm}$; (b) Fluorescence of dispersed DUT-5-NH2 (constructed by $\mathrm{H}_{2}$ BPDC-NH 2 ) and MOF-253 (constructed by $\mathrm{H}_{2} \mathrm{BPyDC}$ ligands) in aqueous solution (30 ppm) when excited at $305 \mathrm{~nm}$.

\section{2- Fluorescence discussion}

As shown in Figure S16, both $\mathrm{H}_{2} \mathrm{BPyDC}$ ligands and MOF-253 showed very weak fluorescence, while $\mathrm{H}_{2} \mathrm{BPDC}-\mathrm{NH}_{2}$ and DUT-5-NH 2 were highly emissive. Therefore, the multivariate BPyDC@MOF-253- $\mathrm{NH}_{2}$ materials only have single emission although the presence of $\mathrm{BPyDC}^{2-}$ ligands in the frameworks. Thus, the second emissive center should be introduced to realize dual-emission.

Through post-synthesis, Eu(III) was anchored in the bpy units in $\mathrm{BPyDC}^{2-}$ ligands to introduce the second emitting. Although the electronic transitions are forbidden by Laporte selection rules for lanthanide cations, strong characteristic red emission were observed in Eu@MOF-253 through antenna effect. The antenna process is similar with that of other lanthanide MOFs. ${ }^{1-2}$ Brifly, BPyDC ${ }^{2-}$ ligands adsorbs photons and was excited to single state. Through intersystem crossing procedure, the electrons reach to the triplet state. Subsequently, the triplet state that matching well with the energy levels of $\mathrm{Eu}(\mathrm{III})$ can sensitize $\mathrm{Eu}$ (III) cations and realize the strong emission. 
Table S1. ICP results of Eu/BPyDC@MOF-253-NH2.

\begin{tabular}{cccc}
\hline Samples & $\mathrm{Al}(\mathrm{wt} \%)$ & $\mathrm{Eu}(\mathrm{wt} \%)$ & $m_{\mathrm{Eu}} / m_{\mathrm{Al}}$ \\
\hline 10-0-Eu/BPyDC@MOF-253-NH 2 & 6.25 & 9.60 & 27.28 \\
9-1-Eu/BPyDC@MOF-253-NH 2 & 6.97 & 7.31 & 18.63 \\
5-5-Eu/BPyDC@MOF-253-NH 2 & 5.35 & 2.14 & 7.10 \\
3-7-Eu/BPyDC@MOF-253-NH 2 & 7.60 & 2.87 & 6.70 \\
1-9-Eu/BPyDC@MOF-253-NH 2 & 7.77 & 2.96 & 6.76 \\
\hline
\end{tabular}

Table S2. Comparison of MOF-based sensors for AA detection.

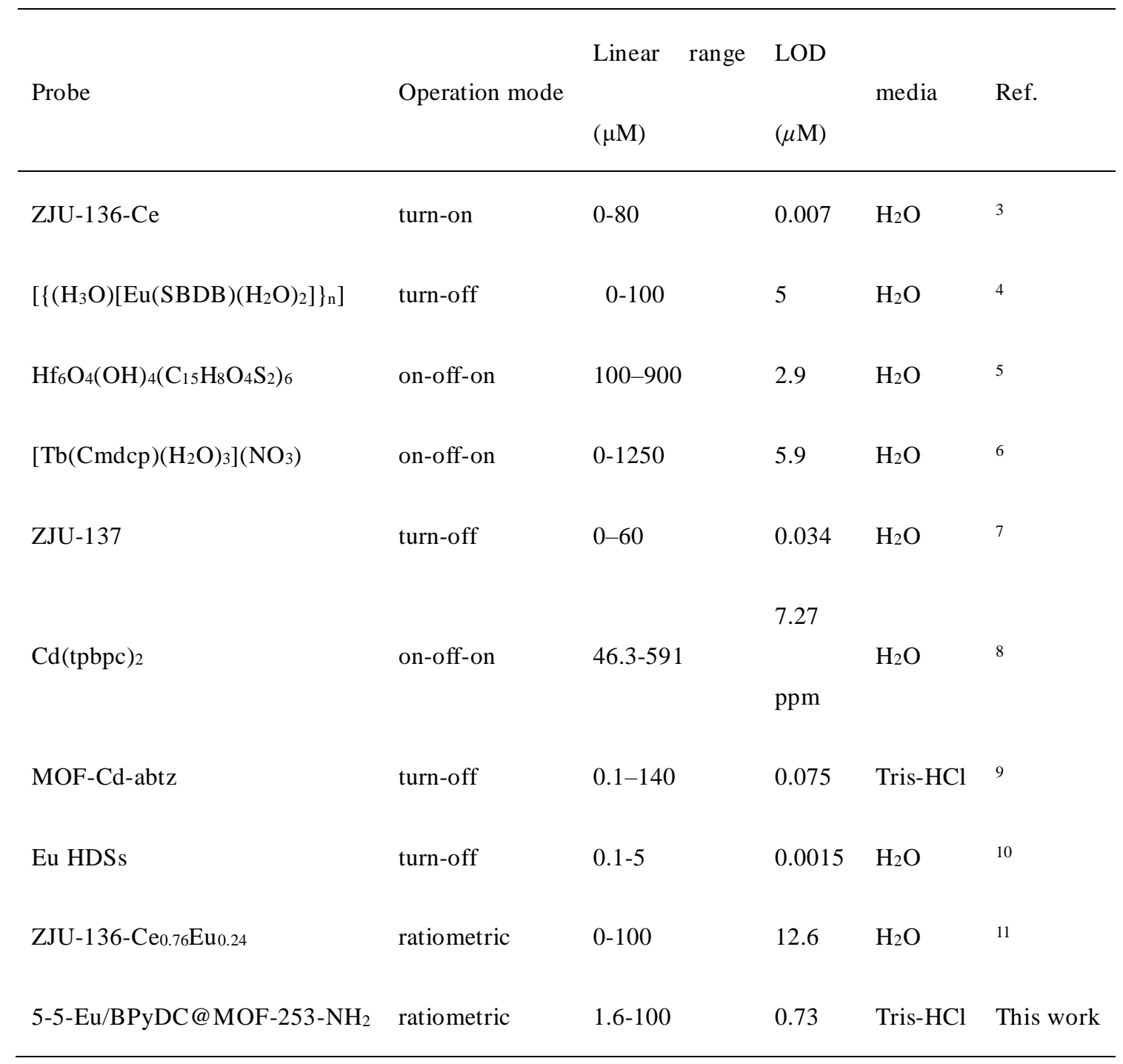


Table S3. Fluorescent lifetimes of 5-5-Eu/BPyDC@ MOF-253-NH2 suspensions with various $\mathrm{NaClO}$ content.

\begin{tabular}{cccccc}
\hline Sample & $\tau_{1}(\mathrm{~s})$ & $\mathrm{Rel}(\%)$ & $\tau_{2}(\mathrm{~s})$ & $\mathrm{Rel}(\%)$ & $\tau(\mathrm{s})$ \\
\hline Suspension $+0 \mu \mathrm{M} \mathrm{ClO}^{-}$ & $7.30 \mathrm{E}-10$ & 13.95 & $8.15 \mathrm{E}-09$ & 86.05 & $7.11 \mathrm{E}-09$ \\
Suspension $+2 \mu \mathrm{M} \mathrm{ClO}^{-}$ & $6.92 \mathrm{E}-10$ & 15.87 & $8.13 \mathrm{E}-09$ & 84.13 & $6.95 \mathrm{E}-09$ \\
Suspension $+10 \mu \mathrm{M} \mathrm{ClO}^{-}$ & $6.37 \mathrm{E}-10$ & 18.85 & $8.04 \mathrm{E}-09$ & 81.15 & $6.64 \mathrm{E}-09$ \\
Suspension $+20 \mu \mathrm{M} \mathrm{ClO}^{-}$ & $6.23 \mathrm{E}-10$ & 23.63 & $8.00 \mathrm{E}-09$ & 76.37 & $6.25 \mathrm{E}-09$ \\
Suspension $+30 \mu \mathrm{M} \mathrm{ClO}^{-}$ & $5.79 \mathrm{E}-10$ & 47.76 & $7.39 \mathrm{E}-09$ & 52.24 & $4.14 \mathrm{E}-09$ \\
\hline
\end{tabular}

Table S4. Fluorescent lifetimes of 5-5-Eu/BPyDC@MOF-253- $\mathrm{NH}_{2}$ suspensions with $30 \mu \mathrm{M}$ of $\mathrm{NaClO}$ after adding various amounts of AA.

\begin{tabular}{llllll}
\hline Sample & $\tau_{1}(\mathrm{~s})$ & $\operatorname{Rel}(\%)$ & $\tau_{2}(\mathrm{~s})$ & $\operatorname{Rel}(\%)$ & $\tau(\mathrm{s})$ \\
\hline Suspension+0 $\mu \mathrm{L} 1 \mathrm{mM}$ AA & $5.79 \mathrm{E}-10$ & 47.76 & $7.39 \mathrm{E}-09$ & 52.24 & $4.14 \mathrm{E}-09$ \\
Suspension+100 $\mu \mathrm{L} 1 \mathrm{mM}$ AA & $5.82 \mathrm{E}-10$ & 33.30 & $7.89 \mathrm{E}-09$ & 66.70 & $5.46 \mathrm{E}-09$ \\
Suspension+200 $\mu \mathrm{L} \mathrm{1}$ mM AA & $6.39 \mathrm{E}-10$ & 17.89 & $8.16 \mathrm{E}-09$ & 82.11 & $6.81 \mathrm{E}-09$ \\
Suspension+300 $\mu \mathrm{L} \mathrm{1} \mathrm{mM} \mathrm{AA}$ & $6.32 \mathrm{E}-10$ & 12.90 & $8.08 \mathrm{E}-09$ & 87.10 & $7.12 \mathrm{E}-09$ \\
\hline
\end{tabular}

1. Cui, Y.; Chen, F.; Yin, X.-B., A ratiometric fluorescence platform based on boric-acid-functional Eu-MOF for sensitive detection of $\mathrm{H}_{2} \mathrm{O}_{2}$ and glucose. Biosens. Bioelectron. 2019, 135, 208-215. 
2. Yang, Z.-R.; Wang, M.-M.; Wang, X.-S.; Yin, X.-B., Boric-Acid-Functional Lanthanide Metal-Organic Frameworks for Selective Ratiometric Fluorescence Detection of Fluoride Ions. Anal. Chem. 2017, 89, 1930-1936.

3. Yue, D.; Zhao, D.; Zhang, J.; Zhang, L.; Jiang, K.; Zhang, X.; Cui, Y.; Yang, Y.; Chen, B.; Qian, G., A luminescent cerium metal-organic framework for the turn-on sensing of ascorbic acid. Chem. Commun. 2017, 53, 11221-11224.

4. Yuan, Y.-Y.; Yang, S.-L.; Zhang, C.-X.; Wang, Q.-L., A new europium metal-organic framework with both high proton conductivity and highly sensitive detection of ascorbic acid. CrystEngComm 2018, 20, 6989-6994.

5. Dalapati, R.; Biswas, S., Aqueous Phase Sensing of $\mathrm{Fe}^{3+}$ and Ascorbic Acid by a Metal-Organic Framework and Its Implication in the Construction of Multiple Logic Gates. Chem. - An Asian J. doi:10.1002/asia.201900546.

6. Wu, K.-Y.; Qin, L.; Fan, C.; Cai, S.-L.; Zhang, T.-T.; Chen, W.-H.; Tang, X.-Y.; Chen, J.-X., Sequential and recyclable sensing of $\mathrm{Fe}^{3+}$ and ascorbic acid in water with a terbium(III)-based metal-organic framework. Dalton Trans. 2019, 48, 8911-8919.

7. Yue, D.; Huang, Y.; Zhang, J.; Zhang, X.; Cui, Y.; Yang, Y.; Qian, G., A Two-Dimensional Metal-Organic Framework as a Fluorescent Probe for Ascorbic Acid Sensing. Eur. J. Inorg. Chem. 2018, 2018, 173-177.

8. Xiao, J.; Liu, J.; Liu, M.; Ji, G.; Liu, Z., Fabrication of a Luminescence-Silent System Based on a Post-Synthetic Modification Cd-MOFs: A Highly Selective and Sensitive Turn-on Luminescent Probe for Ascorbic Acid Detection. Inorg. 
Chem. 2019, 58, 6167-6174.

9. Guo, C.; Jin, Q.; Wang, Y.; Ding, B.; Li, Y.; Huo, J.; Zhao, X., Developing a unique metal-organic framework- $\left\{\left[\mathrm{Cd}(\mathrm{abtz})_{2}(\mathrm{NCS})\right] \cdot\left(\mathrm{ClO}_{4}\right)\right\} \mathrm{n}$ (abtz=1-(4-aminobenzyl)-1,2,4-triazole) as fluorescent probe for highly selective and sensitive detection of ascorbic acid in biological liquid. Sensor Actuat. B-Chem. 2016, 234, 184-191.

10. Li, A.; Zhang, J.; Sun, S.; Ding, E.; Chen, F.; He, S.; Li, T.; Liu, S.; Wang, B., Anion-exchangeable modulated fluorescence strategy for sensitive ascorbic acid detection with luminescent Eu hydroxy double salts nanosunflowers derived from MOFs. Sensor Actuat. B-Chem. 2019, 296, 126636.

11. Yue, D.; Huang, Y.; Zhang, L.; Jiang, K.; Zhang, X.; Cui, Y.; Yu, Y.; Qian, G., Ratiometric luminescence sensing based on a mixed $\mathrm{Ce} / \mathrm{Eu}$ metal-organic framework. J. Mater. Chem. C 2018, 6, 2054-2059. 